Rev. Biol. Trop. 52(1): 119-132, 2004

www.ucr.ac.cr www.ots.ac.cr www.ots.duke.edu

\title{
Recolecta de artrópodos para prospección de la biodiversidad en el Área de Conservación Guanacaste, Costa Rica
}

\author{
Vanessa Nielsen ${ }^{1,2}$, Priscilla Hurtado ${ }^{1}$, Daniel H. Janzen ${ }^{3}$, Giselle Tamayo ${ }^{1} \&$ Ana Sittenfeld ${ }^{1,4}$ \\ 1 Instituto Nacional de Biodiversidad (INBio), Santo Domingo de Heredia, Costa Rica. \\ 2 Dirección actual: Escuela de Biología, Universidad de Costa Rica, 2060 San José, Costa Rica. \\ 3 Department of Biology, University of Pennsylvania, Philadelphia, USA. \\ 4 Dirección actual: Centro de Investigación en Biología Celular y Molecular, Universidad de Costa Rica. \\ vnielsen@cariari.ucr.ac.cr, phurtado@inbio.ac.cr, gtamayo@inbio.ac.cr, djanzen@sas.upenn.edu, sitten@ racsa.co.cr
}

Recibido 21-I-2003. Corregido 19-I-2004. Aceptado 04-II-2004.

\begin{abstract}
This study describes the results and collection practices for obtaining arthropod samples to be studied as potential sources of new medicines in a bioprospecting effort. From 1994 to 1998, 1800 arthropod samples of 6-10 g were collected in 21 sites of the Área de Conservación Guancaste (A.C.G) in Northwestern Costa Rica. The samples corresponded to 642 species distributed in 21 orders and 95 families. Most of the collections were obtained in the rainy season and in the tropical rainforest and dry forest of the ACG. Samples were obtained from a diversity of arthropod orders: $49.72 \%$ of the samples collected corresponded to Lepidoptera, $15.75 \%$ to Coleoptera, $13.33 \%$ to Hymenoptera, $11.43 \%$ to Orthoptera, $6.75 \%$ to Hemiptera, $3.20 \%$ to Homoptera and $7.89 \%$ to other groups. Different life stages per arthropod species were obtained in most samples, $54.26 \%$ of them were adults, $19.90 \%$ corresponded to larvae, $6.46 \%$ to pupae, $6.12 \%$ to pre-pupae, $2.07 \%$ to nymphs and $3.74 \%$ to other stages. Other materials associated to insects like frass represented $11.20 \%$ of the samples collected. Several collecting methods were explored, based on the possibility of accessing the necessary amount of material causing the less impact. Most of the samples were obtained by manual collection (44.38\%), followed by insects breeding (25.73\%), light traps (18.80\%), different types of nets $(10.52 \%)$ and other methods $(0.16 \%)$. In general, collecting methods and practices excluded the use of solvents, mixing different species or life stages in the same bag, which might have introduced undesirable effects in the screening systems for new compounds. Based on the possibility of finding new chemicals in similar samples associated to one arthropod species, the collecting strategy included the generation of several samples from same species, separated according to differences in life stages, collecting sites, ecosystems, seasons, feeding materials or behavioral aspects. This strategy allowed the generation a larger number of samples submitted to bioassays in different areas of pharmaceutical research.
\end{abstract}

Key words: bioprospecting, arthropods, insects, Guanacaste Conservation Area, Costa Rica, collecting methods, Santa Rosa, Guanacaste, Rincón de la Vieja, National Parks.

Los avances de la biotecnología, la robótica, la informática y la biología molecular ofrecen nuevas oportunidades para la bioprospección que pueden adicionalmente estar congruentes con los lineamientos de la Convención de Biodiversidad (Ten Kate 2002).

La bioprospección es una actividad de naturaleza exploratoria, no destructiva, en la que a través de la investigación científica, se pretende la obtención información útil derivada de la recolecta de cantidades mínimas de material biológico-genético, para lograr una aplicación de uso en medicina, agricultura y la industria (Sittenfeld y Lovejoy 1996, Sittenfeld 1999, Setzer et al. 2003). Ejemplos de bioprospección son la búsqueda de nuevos 
compuestos químicos a partir de plantas, microorganismos u otros seres vivos para su uso farmacéutico o agrícola (Sittenfeld 1996).

Tradicionalmente, el énfasis en la búsqueda de nuevos productos naturales para su uso en medicina se ha centrado en plantas, microorganismos y organismos marinos (Tamayo et al. 1997, Demain 1998). A pesar de que los artrópodos son un grupo con alta diversidad biológica, han recibido poca atención como fuente potencial de nuevos productos farmacéuticos. Lo anterior es debido a dificultades en la recolecta, la identificación taxonómica, el reducido conocimiento sobre su historia natural y la escasa información disponible sobre el análisis químico y farmacológico para la detección de compuestos de interés (Sittenfeld $e t$ al. 1999). Los insectos producen y utilizan una gran diversidad de sustancias activas, entre ellas, feromonas, compuestos de defensa y reguladores de crecimiento y diferenciación. Se ha observado gran variabilidad en las sustancias defensivas, tanto a nivel inter-específico como intra-específico. Asimismo, estas sustancias pueden variar dependiendo del sexo, edad, casta, estadio, raza, estación del año, población o la evolución del mecanismo de secreción, además de la dieta de los individuos, lo cual adiciona aún mayor variabilidad a su química (Barboza y Letourneau 1988, Weiss y Eisner 1998). En algunos casos, la producción de sustancias activas derivan de la interacción con microorganismos, como es el caso de la producción de feromonas producidas por bacterias que habitan en el intestino de insectos (Moreno 1997, Dillon et al. 2000, Stipeller et al. 2000, Sittenfeld et al. 2002).

Se estima que en Costa Rica existen aproximadamente 366000 especies de artrópodos, de las cuales, 67000 son conocidas (Obando 2002). En particular, Coleoptera, Lepidoptera, Hymenoptera y Diptera son los órdenes con mayor número de especies (De La Fuente 1994), lo que podría significar una gran diversidad de nuevos compuestos de utilidad.

La búsqueda de nuevos productos farmacéuticos a partir de insectos y otros artrópodos, comprende la recolecta del material biológico y la producción de extractos químicos a partir de los artrópodos, sus partes o materiales asociados. Posteriormente, estos extractos son analizados en ensayos biológicos que permiten detectar nuevas actividades biológicas. Los principios bioactivos son purificados y caracterizados a partir del fraccionamiento guiado por bioensayo (Sittenfeld et al. 1999, Mateo et al. 2001). En el caso de compuestos de interés farmacéutico, estos son analizados por su potencial tóxico y sus aplicaciones clínicas en un proceso que puede tomar más de 10 años y con un costo de cientos de millones de dólares (Thayer 1998, Mateo et al. 2001). Este trabajo describe los resultados y los aspectos prácticos relacionados con la recolecta de artrópodos para la bioprospección, como parte del programa de Grupos Colaborativos Internacionales en Biodiversidad (Rosenthal 2002).

\section{MATERIALES Y MÉTODOS}

La recolecta de muestras de artrópodos se realizó entre 1994 y 1998 (hasta mayo), durante todos los meses del año, en el Área de Conservación Guanacaste (ACG), ubicada en el noroeste de Costa Rica (http://www.acguanacaste.ac.cr). El ACG cubre gran parte de un rectángulo definido por $10^{\circ} 44^{\prime}$ y $11^{\circ} 06^{\prime}$ latitud $\mathrm{N}$ por $85^{\circ} 15^{\prime}$ y $86^{\circ} 00^{\prime}$ longitud oeste (Janzen 1998) y tiene una extensión de 110000 ha en la sección terrestre y 43000 ha en la marina que alberga una gran diversidad de organismos y ecosistemas que varían desde bosque seco, bosque húmedo del Pacífico hasta bosque nuboso en las tierras altas y bosque lluvioso del Atlántico. Las muestras se recolectaron en 21 localidades que incluyen la mayoría de los ecosistemas del ACG.

Las muestras recolectadas se analizaron en las siguientes áreas terapéuticas: enfermedades parasitarias e infecciosas, cáncer, enfermedades del sistema nervioso central y cardiovascular, dermatología e inmunología (Sittenfeld et al. 1999).

Se consideró una muestra de artrópodos como un grupo de individuos de la misma 
especie, estadio y sexo, que provenía de la misma localidad y cuyo peso fresco mínimo estaba entre 6 y $10 \mathrm{~g}$. Materiales asociados como estiércol, panales o mudas fueron también considerados como muestras. Estudios preliminares estimaron que este peso era apropiado para el análisis químico y los ensayos biológicos (Sittenfeld et al.1999). La muestra podía ser de una ecoespecie, que es un grupo de individuos de la misma especie y estadio de vida (adulto o inmaduro), o sexo, alimentándose de alguna otra de las plantas hospederas, o recolectados en otro sitio ecológicamente diferente. Por lo tanto, grupos de artrópodos adultos de la misma especie recolectados en localidades diferentes se consideraron como muestras distintas cuando los sitios presentaban diferencias altitudinales, climáticas, faunísticas y florísticas. De la misma manera, se estableció que si grupos de artrópodos inmaduros de la misma especie y estadio se encontraban en plantas hospederas distintas, serían analizados como muestras separadas. Para cada muestra de artrópodos se conservaron varios testigos de referencia, ya sea en colección húmeda (en alcohol 70\% y constituida principalmente por individuos inmaduros) o montados en alfiler en el caso de los adultos. Esta colección de referencia forma parte del Inventario de Biodiversidad del Instituto Nacional de Biodiversidad (INBio), Santo Domingo de Heredia, Costa Rica.

El muestreo se basó en la disponibilidad de artrópodos en tiempo y espacio en un sitio determinado. La cantidad de tiempo invertida por localidad fue variable, se enfatizó en la recolecta de artrópodos comunes y abundantes en las localidades de estudio. La recolecta se basó en la obtención de muestras en diferentes ecosistemas y zonas de vida, para tener ampliar las posibilidades de localizar muestras con características químicas potencialmente distintas.

Para cada muestra se registró la información de la recolecta y comentarios sobre historia natural y ecología del artrópodo al momento de la recolecta.

Los artrópodos se recolectaron en el campo en bolsas plásticas separadas, una bolsa por sexo. Luego se verificó la identificación taxonómica, el sexo y el estadio. Después se transfirieron a recipientes de vidrio debidamente etiquetados y se almacenaron en congeladores $\left(-20^{\circ} \mathrm{C}\right)$ mientras se completaba el peso final de la muestra. No se recolectó de nuevo una muestra excepto cuando resultó positiva en algún bioensayo.

Se seleccionaron aquellos métodos de recolecta en los cuales no se mezclan especies o sexos durante la captura, ya que algunos insectos cuando son perturbados tienden a producir sustancias defensivas que "contaminan" a todo el grupo, impidiendo la separación posterior de especies o sexos. No se usaron métodos de recolecta tradicionales que utilizan solventes químicos como parte de la captura. Los métodos de recolecta utilizados fueron los siguientes: recolecta manual, trampa de luz, crianza de artrópodos, trampa de foso, red de golpe, red acuática y red aérea (Nielsen 2003). Uno de los métodos de crianza utilizados, consistió en la localización de grupos de larvas en el campo, que fueron cubiertos con una malla, para evitar la depredación por pájaros. Cada grupo se revisó periódicamente para obtener muestras de diferentes estadios.

\section{RESULTADOS Y DISCUSIÓN}

Se recolectó un total de 1800 muestras de artrópodos de 642 especies distribuidas en 21 órdenes y 95 familias. El $96.6 \%$ de las muestras fueron insectos y el $3.4 \%$ otros grupos de artrópodos como Araneae, Iulida, Opiliones, Polydesmida y Amblypygi. En el 75\% de las recolectas, se obtuvo el peso fresco requerido de una muestra. Un total de 1357 muestras alcanzaron la cantidad de extracto necesaria para ser analizadas en uno o varios bioensayos. Los sistemas de muestreo seleccionados fueron adecuados para la generación de muestras de artrópodos para procesos de bioprospección. En general, el tipo de muestreo utilizado permitió la exploración de diferentes sitios, tipos de clima, métodos de recolecta y principalmente la disponibilidad de artrópodos. Esto 
ofreció la oportunidad de escoger y/o cambiar la duración de la recolecta por localidad y movilizarse a otros sitios con mayor cantidad de artrópodos, situación que se hubiera dificultado con otro tipo de muestreo tal como el sistemático. Lo que ayuda a disminuir la interferencia de las dinámica de las poblaciones naturales.

En promedio se recolectaron dos muestras por especie de artrópodo ( $\mathrm{s}=3.32, \mathrm{~N}=578)$. En 372 casos, se recolectó una muestra por especie; en 98, 32 y 24 casos se generaron dos, tres y cuatro muestras de la misma especie, respectivamente. La duración promedio de la recolecta de una muestra fue de 12 días.

En Santa María (bosque lluvioso) se obtuvieron 231 especies, las cuales produjeron 421 muestras diferentes. En el bosque seco (Santa Rosa) se recolectaron 110 especies que generaron 172 muestras, seguida por muestras obtenidas en el bosque húmedo, Cacao con 98 especies y 203 muestras y Pitilla con 67 especies y 79 muestras. Otras localidades en los ecosistemas de transición de seco - húmedo como Góngora generaron 63 especies y un total de 100 muestras. El Sector Santa María se caracteriza por ser accesible durante todo el año. Además, tiene una alta disponibilidad, diversidad y abundancia de artrópodos. Es posible que esto se deba a la influencia que ejercen ambas vertientes y a que la estación lluviosa es más prolongada.

El número de muestras de artrópodos recolectadas varió a través del año. La mayor cantidad de muestras se recolectó en la estación lluviosa (Fig. 1), cuando hay una mayor disponibilidad de recursos alimenticios para los artrópodos y por ende, una alta aparición de organismos. Son frecuentes las emergencias explosivas de órdenes como Lepidoptera y Coleoptera. En las tierras altas del ACG (Pailas, Santa María, Cacao, Góngora, Orosí, Pitilla, San Ramón, San Cristóbal, Teodoro, Campana, Pasmompa y San Josecito), la estación lluviosa se extiende desde finales de abril hasta enero, mientras que en las tierras bajas del ACG (Santa Rosa, Centeno, Jenny, Naranjo, Horizontes, Murciélago, El Hacha, Los Almendros y $\mathrm{El} \mathrm{Amo)} \mathrm{se} \mathrm{inicia} \mathrm{a} \mathrm{finales} \mathrm{de} \mathrm{mayo} \mathrm{y}$ finaliza en noviembre.
Se registraron variaciones en la recolecta de artrópodos en los diferentes meses y años del estudio. Durante 1994 se presentó un rango de alta aparición de artrópodos (meses en donde se recolectaron individuos de especies con potencial de obtener muestras entre 6 y 10 g) entre mayo y julio, y dos picos de recolecta (mes en donde se inició la mayor cantidad de muestras): uno en junio y otro en octubre, (Fig. 1). Durante junio de este año, los órdenes de insectos más recolectados fueron: Lepidoptera, Coleoptera y Orthoptera, y en octubre: Lepidoptera, Orthoptera e Hymenoptera. En 1995 también se presentó un rango de alta aparición entre mayo y julio. El mes donde se recolectaron más artrópodos fue en junio, siendo los principales grupos: Orthoptera, Lepidoptera y Coleoptera. Para el año 1996, se registra el número más alto de recolectas y dos rangos de alta aparición: uno de abril a junio y otro de setiembre a octubre. En abril y setiembre se dieron dos picos de recolecta; en ambos casos los grupos más recolectados fueron: Lepidoptera, Orthoptera y Coleoptera. Durante 1997 se dieron dos picos de alta aparición: uno de abril a junio y otro de octubre a noviembre. Mayo y octubre fueron los meses donde se recolectó la mayor cantidad de muestras; en mayo los principales grupos fueron: Lepidoptera, Hemiptera y Coleoptera, mientras que en octubre fueron: Lepidoptera, Coleoptera y Orthoptera.

Los promedios mensuales de precipitación de Santa Rosa (tierras bajas) y Orosí (tierras intermedias del ACG), más altos coinciden con las mayores cantidades de artrópodos recolectadas.

En 1997 se recolectaron 266 muestras de insectos, siendo el año en que hubo la menor cantidad de recolectas, debido a que el área de trabajo se limitó a las zonas bajas del ACG, ya que se trató de recolectar artrópodos que se alimentaban de frutos de árboles del bosque seco. Generalmente, eran artrópodos muy pequeños, por lo que fue difícil obtener muestras con un mínimo de $5 \mathrm{~g}$. Otra causa del bajo número de muestras fue la precipitación, ya que este año se informa como uno de los más secos desde 1983 (debido al Fenómeno del Niño) y con las 


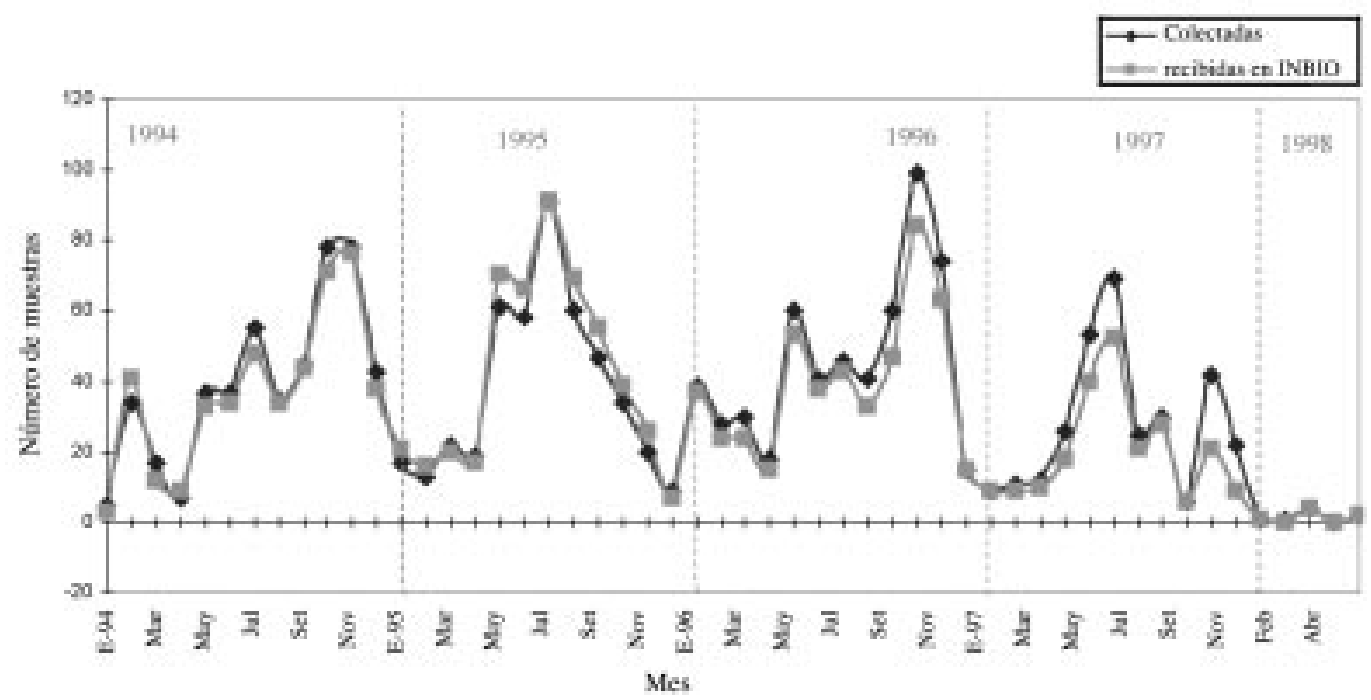

Fig. 1. Muestras de artrópodos recolectadas en el Área de Conservación Guanacaste y entregadas al laboratorio del INBio, de enero de 1994 a mayo de 1998.

Fig. 1. Samples of collected artropods in Guanacaste Conservation Area and delivered to INBio Laboratory, from 1994 January to 1998 May.

densidades de insectos más bajas que se han informado en toda la historia de la investigación del ACG (Sittenfeld et al. 1999). En 1997, el mes donde se recolectó la mayor cantidad de muestras fue junio $(n=43)$, las cuales correspondieron principalmente a artrópodos de los grupos Lepidoptera y Coleoptera.

En los meses de mayor aparición de artrópodos durante este estudio, se recolectaron principalmente individuos de los grupos Lepidoptera, Orthoptera y Hemiptera. La aparición de estos grupos coincide con el inicio de las lluvias y el consecuente aumento en la disponibilidad de vegetación y por ende de alimento para órdenes como: Orthoptera, Hemiptera, Homoptera, Lepidoptera, cuyos adultos emergen en esta época. Otros grupos que se recolectaron al inicio de la lluvias fueron: Hymenoptera (avispas, abejas) y Polydesmida (milpiés). Hymenoptera fue un grupo muy activo durante la estación lluviosa, ya que hay muchas plantas en floración y sus adultos se alimentan de polen y néctar. Los representantes del orden Polydesmida se alimentan de materia orgánica en descomposición y son también muy abundantes en esta época del año.
El porcentaje de las muestras recolectadas por orden fue el siguiente: Lepidoptera 49.72\%, Coleoptera $15.75 \%$, Hymenoptera $13.33 \%$, Orthoptera $11.43 \%$, Hemiptera $6.75 \%$, Homoptera $3.20 \%$ y otros grupos 7.89\%. En la categoría Otros se encuentran 13 órdenes, entre los principales están: Araneae, Diptera, Amblypygi, Isoptera, Iulida, Neuroptera, Odonata y Thysanoptera, los cuales contribuyeron con el aumento de la variabilidad de muestras. En el orden Lepidoptera se recolectaron 212 especies (572 muestras), en Coleoptera 121 especies (182 muestras), en Hymenoptera 42 especies (154 muestras), y en Orthoptera 63 especies (132 muestras).

El orden Hymenoptera produjo una alta cantidad de muestras, ya que ocasionalmente fue posible separar las partes del panal y sus diferentes estadios.

El porcentaje de las muestras recolectadas por estadio fue el siguiente: adultos $54.26 \%$, larvas $19.90 \%$, estiércol $11.20 \%$, pupa $6.46 \%$, prepupa $6.12 \%$, ninfas $2.07 \%$ y otros estadios $3.74 \%$. Las muestras de estiércol provenían de las bolsas de crianza. 
Las especies de insectos con un mayor número de muestras recolectadas fueron: Rothschildia triloba (Saturniidae) con 32 muestras, Agelaia sp. (Vespidae) con 31, Polybia diguetana (Vespidae) con 30 muestras, Automeris postalbida (Saturniidae) con 27 , lo que indica que es posible obtener un número alto de ecoespecies en el grupo de los artrópodos.

En el Cuadro 1 se incluye la lista de artrópodos recolectados en esta investigación así como las plantas asociadas a cada muestra. Algunos datos corresponden a nuevos informes, tal como Pachygonidia ribbei (Saturniidae), recolectada en el Sector Santa María alimentándose de Hamelia patens (Rubiaceae), la cual fue un nuevo informe de localidad y planta hospedera, al momento de la recolecta. Se identificaron 124 especies de plantas hospederas asociadas con los insectos recolectados, las cuales corresponden a 69 familias diferentes y 145 especies. El $82.50 \%$ de las especies de plantas que se identificaron se informan como hospederas del grupo Lepidoptera. El porcentaje restante correspondió a plantas en las que se encontraron artrópodos de los órdenes: Coleoptera $(9.39 \%)$, Hemiptera $(4.97 \%)$, Homoptera $(1.40 \%)$ y Orthoptera $(0.37 \%)$; principalmente alimentándose de su savia, hojas o flores. Algunas especies de artrópodos también se encontraron copulando en estas plantas.

En el caso de los coleópteros, individuos de las familias Chrysomelidae, Curculionidae, Meloidae y Cerambycidae, se encontraron asociados a árboles, arbustos o hierbas, ya sea alimentándose de su follaje o en el interior de su tronco o tallo.

En los órdenes Hemiptera y Homoptera también se recolectaron organismos herbívoros, como insectos de las familias Membracidae (Homoptera), Aphrophoridae (Homoptera), Pentatomidae (Hemiptera), Coreidae (Hemiptera). Algunas especies de hemípteros son generalistas, como Pharypia pulchella (Pentatomidae) que se recolectó alimentándose en 7 plantas diferentes: Genipa americana (Rubiaceae), $\mathrm{Pi}$ cramnia sp. (Simaroubaceae), Acalypha diversifolia (Euphorbiaceae), Mouriri myrtilloides (Melastomataceae), Randia subcordata
(Rubiaceae), Tetracera volubilis (Dilleniaceae) y Serjania sp. (Sapindaceae). Por otra parte, otros hemípteros como Thasus sp. se encontraron alimentándose de Pithecellobium dulce (Fabaceae/Mimosaceae), mientras que dos miembros de la familia Pentatomidae: Edessa sp. y Ramosiana insignis se recolectaron mientras comian Acalypha diversifolia (Euphorbiaceae) y Schoepfia schreberi (Olacaceae), respectivamente, coincidiendo con la literatura (Slater y Baranowski 1978). Guayaquila gracilicornis (Membracidae) se encontró alimentándose de Spondias sp. (Anacardiaceae), Umbonia crassicornis (Membracidae) comiendo Inga punctata (Fabaceae/Mimosaceae) y Enchophora sanguinea (Fulgoridae) alimentándose de la corteza de Clethra mexicana (Clethraceae) lo cual corresponde con lo informado por Maes (1998). Las ninfas y adultos del orden Orthoptera se les podía encontrar más fácilmente durante los días soleados en áreas abiertas, en parches en regeneración y pastizales cercanos a zonas boscosas.

En algunas plantas se recolectaron varias especies de artrópodos, tales como: Inga punctata (Fabaceae/Mim.) en la cual habían 7 especies de artrópodos; Enterolobium cyclocarpum (Fabaceae/Mim.) y Quercus oleoides (Fagaceae) con 6 especies en cada una y Persea americana (Lauraceae), Cordia alliodora (Boraginaceae), Guazuma ulmifolia (Sterculiaceae) y Prunus annularis (Rosaceae) con 5 especies.

Los métodos de recolecta de artrópodos más eficaces fueron aquellos que permitieron obtener muestras mayores de $5 \mathrm{~g}$ en el menor tiempo posible. El método más utilizado fue la recolecta manual $(44.38 \%)$, seguido por crianza de insectos $(25.73 \%)$, trampa de luz (18.80\%), diferentes tipos de redes $(10.52 \%)$ y otros métodos como la trampa de foso $(0.16 \%)$. Las especies de artrópodos que alcanzaron el peso mínimo requerido para prospección presentaron una o varias de las siguientes características: eran abundantes, tendían a agruparse para alimentarse, reproducirse o simplemente posarse sobre alguna planta. 
CUADRO 1

Asociaciones planta-insecto identificadas durante el período de estudio en el Área de Conservación Guanacaste (Costa Rica)

TABLE 1

Plant-insect association finded during the study period in Guanacaste Conservation Area (Costa Rica)

\begin{tabular}{|c|c|c|c|c|}
\hline Familia de planta & Especie de planta & Orden de insecto & Familia de insecto & Especie de insecto \\
\hline Acanthaceae & Aphelandra scabra & Lepidoptera & Nymphalidae & Chlosyne poecile \\
\hline Acanthaceae & Aphelandra sp. & Lepidoptera & Nymphalidae & Chlosyne melanarge \\
\hline Acanthaceae & Justicia sp. & Lepidoptera & Hesperiidae & Celaenorrhinus eligius \\
\hline \multirow[t]{2}{*}{ Anacardiaceae } & Astronium graveolens & Lepidoptera & Limacodidae & Acharia ophelians \\
\hline & & Coleoptera & Cerambycidae & $\begin{array}{l}\text { Brasilianus mexicanus } \\
\text { Parandra glabra }\end{array}$ \\
\hline Anacardiaceae & Mangifera indica & Lepidoptera & Limacodidae & Acharia ophelians \\
\hline \multirow[t]{3}{*}{ Anacardiaceae } & Spondias mombin & Lepidoptera & Limacodidae & Acharia ophelians \\
\hline & & Coloeptera & Bruchidae & Amblycercus spondiae \\
\hline & & Lepidoptera & Saturniidae & Eacles imperialis \\
\hline Anacardiaceae & Spondias sp. & Hemiptera & Membracidae & Guayaquila gracilicornis \\
\hline Annonaceae & Annona purpurea & Lepidoptera & Saturniidae & Hylesia sp. \\
\hline Annonaceae & Annona reticulata & Lepidoptera & Sphingidae & Cocytius antaeus \\
\hline Annonaceae & Guatteria sp. & Lepidoptera & Saturniidae & Automeris postalbida \\
\hline Annonaceae & Guatteria tonduzii & Lepidoptera & Sphingidae & Cocytius lucifer \\
\hline Annonaceae & Rollinia membranacea & Lepidoptera & Papilionidae & Eurytides euryleon \\
\hline Apocynaceae & Forsteronia spicata & Lepidoptera & Sphingidae & Pachylioides resumens \\
\hline Araliaceae & Dendropanax sp. & Lepidoptera & Nymphalidae & Hamadryas amphinome \\
\hline Araliaceae & Dendropanax sp. & Lepidoptera & Saturniidae & Rothschildia triloba \\
\hline Arecaceae & Acrocomia aculeata & Lepidoptera & Nymphalidae & Opsiphanes cassina \\
\hline Aristolochiaceae & Aristolochia sp. & Lepidoptera & Papilionidae & Battus belus \\
\hline Aristolochiaceae & Aristolochia sp. & Lepidoptera & Papilionidae & Parides sesostris \\
\hline Asclepiadaceae & Asclepias curassavica & Lepidoptera & Nymphalidae & Danaus plexippus \\
\hline Asteraceae & Clibadium sp. & Coleoptera & Curculionidae & Lixus sp. \\
\hline Asteraceae & Lasianthaea fruticosa & Lepidoptera & Nymphalidae & Eueides procula \\
\hline Asteraceae & Liabum bourgeaui & Lepidoptera & Nymphalidae & Actinote leucomelas \\
\hline Asteraceae & Salmea scandens & Lepidoptera & Nymphalidae & Tegosa anieta \\
\hline \multirow[t]{2}{*}{ Asteraceae } & Vernonia patens & Homoptera & Aphrophoridae & Cephisus sp. \\
\hline & & Coleoptera & Curculionidae & Exophthalmus sp. \\
\hline \multirow[t]{2}{*}{ Bignoniaceae } & Godmania aesculifolia & Hemiptera & Coreidae & Acanthocepha \\
\hline & & & Pentatomidae & Edessa sp. \\
\hline Bignoniaceae & Tabebuia ochraceae & Lepidoptera & Papilionidae & Heraclides anchesiadess \\
\hline \multirow[t]{2}{*}{ Bignoniaceae } & Tabebuia rosea & Lepidoptera & Crambidae & Eulepte sp. \\
\hline & & & Lymantriidae & Phiditia sp. \\
\hline \multirow[t]{3}{*}{ Bombacaceae } & Bombacopsis quinata & Lepidoptera & Saturniidae & Arsenura armida \\
\hline & & & & Caio championi \\
\hline & & & & Titaea tamerlan \\
\hline \multirow[t]{3}{*}{ Boraginaceae } & Cordia alliodora & Lepidoptera & Saturniidae & Automeris zugana \\
\hline & & & Saturniidae & Manduca corallina \\
\hline & & & Sphingidae & Manduca sp. \\
\hline Boraginaceae & Cordia dentata & Coleoptera & Cantharidae & Chauliognathus tricolor \\
\hline Boraginaceae & Cordia sp. & Coleoptera & Chrysomelidae & Ischnocodia annulus \\
\hline \multirow[t]{2}{*}{ Burseraceae } & Bursera simarouba & Lepidoptera & Saturniidae & Eacles imperialis \\
\hline & & & & Hylesia sp. \\
\hline Cannaceae & Canna sp. & Lepidoptera & Nymphalidae & Caligo sp. \\
\hline Cannaceae & Canna sp. & Lepidoptera & Hesperiidae & Calpodes ethilus \\
\hline Cannaceae & Canna sp. & Lepidoptera & Hesperiidae & Rhinthon cubana \\
\hline Capparidaceae & Capparis frondosa & Lepidoptera & Nymphalidae & Perrhybris pyrrha \\
\hline Capparidaceae & Cleome spinosa & Lepidoptera & Pieridae & Ascia monuste \\
\hline Caprifoliaceae & Viburnum costaricanum & Lepidoptera & Saturniidae & Rothschildia triloba \\
\hline
\end{tabular}


CUADRO 1 (continuación...) / TABLE 1 (continued...)

\begin{tabular}{|c|c|c|c|c|}
\hline Familia de planta & Especie de planta & Orden de insecto & Familia de insecto & Especie de insecto \\
\hline Caricaceae & Carica papaya & Lepidoptera & Sphingidae & Erinnys ello \\
\hline Cecropiaceae & Cecropia sp. & Lepidoptera & Noctuidae & Dyops sp. \\
\hline \multirow[t]{2}{*}{ Cecropiaceae } & Coussapoa nymphaefolia & Lepidoptera & Saturniidae & Arsenura sp. \\
\hline & & & Noctuidae & Dyops sp. \\
\hline Cecropiaceae & Pourouma bicolor & Lepidoptera & Saturniidae & Automeris postalbida \\
\hline Chrysobalanaceae & Hirtella racemosa & Lepidoptera & Saturniidae & Automeris postalbida \\
\hline \multirow[t]{2}{*}{ Clethraceae } & Clethra mexicana & Homoptera & Fulgoridae & Enchophora sanguinea \\
\hline & & Lepidoptera & Sphingidae & Eumorpha triangulum \\
\hline \multirow[t]{2}{*}{ Clusiaceae } & Vismia baccifera & Lepidoptera L & Saturniidae & Periphoba arcaei \\
\hline & & & Hesperiidae & Pyrrhopyge zenodorus \\
\hline Convolvulaceae & Ipomoea trifida & Coleoptera & Meloidae & Epicauta pennsylvanica \\
\hline Dilleniaceae & Curatella americana & Lepidoptera & Sphingidae & Enyo ocypete \\
\hline Dilleniaceae & Davilla sp. & Lepidoptera & Sphingidae & Enyo ocypete \\
\hline Dilleniaceae & Doliocarpus sp. & Lepidoptera & Sphingidae & Eumorpha sp. \\
\hline \multirow[t]{2}{*}{ Dilleniaceae } & Tetracera volubilis & Lepidoptera & Sphingidae & Enyo ocypete \\
\hline & & Hemiptera & Pentatomidae & Pharypia pulchella \\
\hline \multirow[t]{4}{*}{ Euphorbiaceae } & Acalypha diversifolia & Lepidoptera & Saturniidae & Automeris postalbida \\
\hline & & Hemiptera & Pentatomidae & Lonomia sp. \\
\hline & & & & Edessa sp. \\
\hline & & & & Pharypia pulchella \\
\hline Euphorbiaceae & Acalypha sp. & Hemiptera & Coreidae & Hypselonotus sp. \\
\hline Euphorbiaceae & Acalvpha sp. & Lepidoptera & Saturniidae & Automeris postalbida \\
\hline Euphorbiaceae & Croton niveus & Coleoptera & Lygaeidae & Oncopeltus sp. \\
\hline Euphorbiaceae & Croton sp. & Lepidoptera & Saturniidae & Hylesia sp. \\
\hline Euphorbiaceae & Dalechampia heteromorpha & Lepidoptera & Nymphalidae & Hamadryas amphinome \\
\hline \multirow[t]{2}{*}{ Euphorbiaceae } & Euphorbia schlechtendalii & Lepidoptera & Doidae & Doa sp. \\
\hline & & & Sphingidae & Erinnys ello \\
\hline Euphorbiaceae & Manihot esculenta & Lepidoptera & Sphingidae & Erinnys ello \\
\hline Euphorbiaceae & Sapium glandulosa c.f. & Lepidoptera & Saturniidae & Rothschildia triloba \\
\hline Fabaceae/caes. & Cassia emarginata & Lepidoptera & Saturniidae & Sysphinix colla \\
\hline Fabaceae/caes. & Cassia fruticosa & Lepidoptera & Pieridae & Eurema xanthochlora \\
\hline Fabaceae/caes. & Delonix regia & Coleoptera & Meloidae & Epicauta mayor \\
\hline Fabaceae/caes. & Hymenaea courbaril & Coleoptera & Curculionidae & Rhinochenus stigma \\
\hline Fabaceae/caes. & Senna hayesiana & Lepidoptera & Pieridae & Phoebis philea \\
\hline \multirow[t]{2}{*}{ Fabaceae/caes. } & Senna papillosa & Lepidoptera & Hesperiidae & Astraptes fulgerator \\
\hline & & & Pieridae & Eurema xanthochlora \\
\hline Fabaceae/mim. & Acacia sp. & Hymenoptera & Formicidae & Pseudomyrmex belti \\
\hline \multirow[t]{3}{*}{ Fabaceae/mim. } & Albizzia adinocephala & Lepidoptera & Noctuidae & Lois fearni \\
\hline & & & Saturniidae & Syssphinix molina \\
\hline & & & & Syssphinix colla \\
\hline \multirow[t]{2}{*}{ Fabaceae/mim. } & Enterolobium cyclocarpum & Lepidoptera & Saturniidae & Molippa nibasa \\
\hline & & Hemiptera & Coreidae & Savius jurgiosus \\
\hline \multirow[t]{6}{*}{ Fabaceae/mim. } & Inga punctata & Lepidoptera & Saturniidae & Adeloneivaia jason \\
\hline & & Lepidoptera & Megalopygidae & Automeris belti \\
\hline & & Lepidoptera & Nymphalidae & Automeris postalbida \\
\hline & & Homoptera & Membracidae & Megalopyge tharops \\
\hline & & & & Morpho polyphemus \\
\hline & & & & Umbonia crassicornis \\
\hline Fabaceae/mim. & Inga sp. & Lepidoptera & Saturniidae & Adeloneivaia jason \\
\hline Fabaceae/mim. & Inga sp. & Lepidoptera & Saturniidae & Automeris postalbida \\
\hline \multirow[t]{2}{*}{ Fabaceae/mim. } & Inga vera & Lepidoptera & Saturniidae & Adeloneivaia jason \\
\hline & & & Notodontidae & \\
\hline Fabaceae/mim. & Pithecellobium dulce & Hemiptera & Coreidae & Thasus sp. \\
\hline Fabaceae/mim. & Pithecellobium longifolium & Lepidoptera & Saturniidae & Adeloneivaia jason \\
\hline
\end{tabular}


CUADRO 1 (continuación...) / TABLE 1 (continued...)

\begin{tabular}{|c|c|c|c|c|}
\hline Familia de planta & Especie de planta & Orden de insecto & Familia de insecto & Especie de insecto \\
\hline Fabaceae/mim. & Pithecelobium valerioi & Lepidoptera & Nymphalidae & Morpho polyphemus \\
\hline \multirow[t]{2}{*}{ Fabaceae/mim. } & Samanea saman & Coleoptera & Bruchidae & Merobruchus columbinus \\
\hline & & Lepidoptera & Saturniidae & Syssphinix colla \\
\hline \multirow[t]{2}{*}{ Fabaceae/pap. } & Ateleia herbert-smithii & Coleoptera & Curculionidae & Apion herbert-smithii \\
\hline & & Lepidoptera & Saturniidae & Eacles imperialis \\
\hline Fabaceae/pap. & Dalbergia retusa & Lepidoptera & Saturniidae & Automeris metzli \\
\hline Fabaceae/pap. & Dioclea megalocarpa & Coleoptera & Bruchidae & Caryedes brasiliensi \\
\hline \multirow[t]{2}{*}{ Fabaceae/pap. } & Gliricia sepium & Homoptera & Aphrophoridae & Cephisus sp. \\
\hline & & Lepidoptera & Saturniidae & Eacles imperialis \\
\hline Fabaceae/pap. & Lonchocarpus acuminatus & Lepidoptera & Saturniidae & Eacles imperialis \\
\hline Fabaceae/pap. & Lonchocarpus minimiflorous & Lepidoptera & Saturniidae & Hylesia sp. \\
\hline \multirow{2}{*}{ Fabaceae/pap. } & Lonchocarpus oliganthus & Lepidoptera & Saturniidae & Automeris postalbida \\
\hline & & & Nymphalidae & Morpho peleides \\
\hline Fabaceae/pap. & Lonchocarpus sp. & Lepidoptera & Nymphalidae & Morpho peleides \\
\hline Fabaceae/pap. & Lonchocarpus sp. & Lepidoptera & Nymphalidae & Morpho sp. \\
\hline Fabaceae/pap. & Machaerium biovulatum & Coleoptera & Chrysomelidae & Oxychalepus alienus \\
\hline \multirow[t]{3}{*}{ Fagaceae } & Quercus oleoides & Lepidoptera & Saturniidae & Automeris metzli \\
\hline & & & & Hylesia sp. \\
\hline & & & & Periphoba arcaei \\
\hline Flacourtiaceae & Casearia arguta & Lepidoptera & Saturniidae & Lonomia sp. \\
\hline \multirow[t]{2}{*}{ Flacourtiaceae } & Casearia corymbosa & Lepidoptera & Hesperiidae & Mysoria ambiguas \\
\hline & & & Saturniidae & Rothschildia lebeau \\
\hline Flacourtiaceae & Hasseltia floribunda & Lepidoptera & Saturniidae & Lonomia sp. \\
\hline Heliconiaceae & Heliconia sp. & & Cercopidae & Mahanarva costaricens \\
\hline Heliconiaceae & Heliconia sp. & Lepidoptera & Hesperiidae & Talides sp. \\
\hline Hippocastanaceae & Billia hippocastanus & Lepidoptera & Saturniidae & Lonomia sp. \\
\hline \multirow[t]{2}{*}{ Lauraceae } & Cinnamomum brenesii & Lepidoptera & Nymphalidae & Archaeoprepona meander \\
\hline & & & Saturniidae & Hylesia sp. \\
\hline Lauraceae & Nectandra salicifolia & Lepidoptera & Saturniidae & Copaxa moinieri \\
\hline Lauraceae & Nectandra sp. & Lepidoptera & Saturniidae & Copaxa moinieri \\
\hline Lauraceae & Nectandra sp. & Lepidoptera & Sphingidae & Adhemarius ypsilon \\
\hline Lauraceae & Nectandra sp. & Lepidoptera & Pyralidae & Deuterollyta subcurvalis \\
\hline Lauraceae & Nectandra sp. & Lepidoptera & Lasiocampidae & Euglyphis deusta \\
\hline Lauraceae & Ocotea sp. & Lepidoptera & Saturniidae & Copaxa moinieri \\
\hline \multirow[t]{2}{*}{ Lauraceae } & Ocotea veraguensis & Lepidoptera & Sphingidae & Adhemarius ypsilon \\
\hline & & Lepidoptera & Saturniidae & Copaxa moinieri \\
\hline \multirow[t]{2}{*}{ Lauraceae } & Persea americana & Lepidoptera & Saturniidae & Copaxa moinieri \\
\hline & & & & Copaxa sp. \\
\hline Malpighiaceae & Byrsonima crassifolia & Lepidoptera & Notodontidae & Bardaxima lucilinea \\
\hline Malvaceae & Hampea appendiculata & Lepidoptera & Saturniidae & Hylesia sp. \\
\hline Malvaceae & Malvaviscus sp. & Lepidoptera & Saturniidae & Arsenura drucei \\
\hline Marantaceae & Calathea sp. & Coleoptera & Lygaeidae & Leptoscelis quadrasigna \\
\hline \multirow[t]{2}{*}{ Melastomataceae } & Conostegia xalapensis & Lepidoptera & Notodontidae & Drugera sp. \\
\hline & & & & Naprepa houla \\
\hline Melastomataceae & Mouriri myrtilloides & Hemiptera & Pentatomidae & Pharypia pulchella \\
\hline \multirow[t]{2}{*}{ Meliaceae } & Guarea glabra & Lepidoptera & Saturniidae & Automeris postalbida \\
\hline & & Lepidoptera & Hesperiidae & Passova gelliass \\
\hline Moraceae & Brosimum alicastrum & Lepidoptera & Saturniidae & Automeris postalbida \\
\hline Moraceae & Ficus cotinifolia & Lepidoptera & Sphingidae & Pachylia sp. \\
\hline Moraceae & Naucleopsis capirensis & Lepidoptera & Sphingidae & Pachylia sp. \\
\hline Musaceae & Musa sp. & Lepidoptera & Nymphalidae & Caligo eurilochus \\
\hline Musaceae & Musa sp. & Lepidoptera & Nymphalidae & Caligo telemonius \\
\hline Musaceae & Musa sp. & Lepidoptera & Nymphalidae & Caligo sp. \\
\hline Myristicaceae & Virola koschnyi & Lepidoptera & Saturniidae & Rhescyntis hippodamia \\
\hline
\end{tabular}


CUADRO 1 (continuación...) / TABLE 1 (continued...)

\begin{tabular}{|c|c|c|c|c|}
\hline Familia de planta & Especie de planta & Orden de insecto & Familia de insecto & Especie de insecto \\
\hline Myrsinaceae & Ardisia revoluta & Lepidoptera & Saturniidae & Periphoba arcaei \\
\hline Myrtaceae & Eugenia hypargyrea & Lepidoptera & Saturniidae & Hylesia lineata \\
\hline Myrtaceae & Myrcia splendens & Lepidoptera & Saturniidae & Automeris banus \\
\hline Myrtaceae & Psidium guajava & Lepidoptera & Saturniidae & $\begin{array}{l}\text { Citheronia sp. } \\
\text { Hylesia } \mathrm{sp.}\end{array}$ \\
\hline Myrtaceae & Psidium guineense & Lepidoptera & $\begin{array}{l}\text { Saturniidae } \\
\text { Mimallonidae }\end{array}$ & $\begin{array}{l}\text { Citheronia sp. } \\
\text { Mimallo amilia }\end{array}$ \\
\hline Nyctaginaceae & Neea sp. & Lepidoptera & Riodinidae & Euselasia sergia \\
\hline Olacaceae & Schoepfia schreberi & Hemiptera & Pentatomidae & Ramosiana insignis \\
\hline Oleaceae & Chionanthus panamensis & Lepidoptera & Saturniidae & Hylesia sp. \\
\hline Phytolaccaceae & Stegnosperma cubense & Othoptera & Romaleidae & Taeniopoda varipennis \\
\hline Piperaceae & Piper sp. & Coleoptera & Curculionidae & Exophthalmus sp. \\
\hline Poaceae & Rottboellia sp. & Lepidoptera & Noctuidae & Mocis repanda \\
\hline \multirow[t]{2}{*}{ Proteaceae } & Roupala montana & Lepidoptera & Mimallonidae & Mimallo amilia \\
\hline & & Coleoptera & Tenebrionidae & Epitragus aurulentus \\
\hline Ranunculaceae & Clematis haenkeiana & Coleoptera & Meloidae & Epicauta mayor \\
\hline Ranunculaceae & Clematis sp. & Coleoptera & Meloidae & Epicauta major \\
\hline Ranunculaceae & Clematis sp. & Lepidoptera & Saturniidae & Hylesia sp. \\
\hline Rhamnaceae & Gouania polygama & Lepidoptera & Notodontidae & Crinodes sp. \\
\hline Rosaceae & Eriobotrya japonica & Hemiptera & Scutelleridae & Augocoris illustris \\
\hline Rosaceae & Prunus annularis & Lepidoptera & Saturniidae & $\begin{array}{l}\text { Hylesia sp. } \\
\text { Lonomia sp. } \\
\text { Rothschildia triloba }\end{array}$ \\
\hline Rubiaceae & Chiococca pachyphylla & Lepidoptera & Saturniidae & Rothschildia triloba \\
\hline Rubiaceae & Exostema mexicanum & Lepidoptera & Saturniidae & Rothschildia triloba \\
\hline Rubiaceae & Genipa americana & Hemiptera & Pentatomidae & Pharypia pulchella \\
\hline Rubiaceae & Hamelia patens & Lepidoptera & Sphingidae & $\begin{array}{l}\text { Pachygonidia ribbei } \\
\text { Xylophanes juanita } \\
\text { Xylophanes sp. }\end{array}$ \\
\hline Rubiaceae & Palicourea padifolia & Lepidoptera & Sphingidae & Xylophanes germen \\
\hline Rubiaceae & Palicourea sp. & Lepidoptera & Sphingidae & Xylophanes \\
\hline Rubiaceae & Pentagonia sp. & Lepidoptera & Saturniidae & Automeris postalbida \\
\hline Rubiaceae & Psychotria elata & Lepidoptera & Sphingidae & Xylophanes germen \\
\hline Rubiaceae & Psychotria horizontalis & Lepidoptera & Sphingidae & Xylophanes juanita \\
\hline Rubiaceae & Randia sp. & Lepidoptera & Oxytenidae & Oxytenia sp. \\
\hline Rubiaceae & Randia subcordata & Hemiptera & Pentatomidae & Pharypia pulchella \\
\hline Rubiaceae & Rondeletia buddleioides & Lepidoptera & Nymphalidae & Adelpha iphiclus \\
\hline Rubiaceae & Spermacoce ovalifolia & Lepidoptera & Sphingidae & Xylophanes sp. \\
\hline Rutaceae & Pilocarpus racemosus & Lepidoptera & Papilionidae & Heraclides anchisiades \\
\hline Sapindaceae & Cupania guatemalensis & Lepidoptera & Saturniidae & Automeris zugana \\
\hline Sapindaceae & Cupania sp. & Lepidoptera & Saturniidae & Automeris postalbida \\
\hline Sapindaceae & Cupania sp. & Lepidoptera & Saturniidae & Lonomia sp. \\
\hline Sapindaceae & Paullinia cururu & Lepidoptera & Notodontidae & Diduga argentilinea \\
\hline \multirow[t]{2}{*}{ Sapotaceae } & Chrysophyllum brenesii & Lepidoptera & Arctiidae & Neonerita dorsipuncta \\
\hline & & & Saturniidae & Periphoba arcaei \\
\hline Sapotaceae & Chrysophyllum sp. & Lepidoptera & Saturniidae & Automeris postalbida \\
\hline Sapotaceae & Manilkara sp. & Lepidoptera & Saturniidae & Copiopteryx semiramis \\
\hline Simaroubaceae & Picramnia sp. & Hemiptera & Pentatomidae & Pharypia pulchella \\
\hline Simaroubaceae & Picrasma excelsa & Lepidoptera & Saturniidae & Rothschildia triloba \\
\hline \multirow[t]{3}{*}{ Solanaceae } & Solanum hayesii & Coleoptera & Chrysomelidae & Colaspis sp. \\
\hline & & & Meloidae & Epicauta carmelita \\
\hline & & & Chrysomelidae & Leptinotarsa undecimlineata \\
\hline Solanaceae & Solanum jamaicense & Lepidoptera & Sphingidae & Manduca sexta \\
\hline Solanaceae & Solanum sp. & Coleoptera & Meloidae & Epicauta major \\
\hline
\end{tabular}


CUADRO 1 (continuación...) / TABLE 1 (continued...)

\begin{tabular}{|c|c|c|c|c|}
\hline Familia de planta & Especie de planta & Orden de insecto & Familia de insecto & Especie de insecto \\
\hline Solanaceae & Solanum suaveolens & Coloeptera & Meloidae & Epicauta major \\
\hline Sterculiaceae & Guazuma ulmifolia & Lepidoptera & Saturniidae & $\begin{array}{l}\text { Arsenura armida } \\
\text { Arsenura batesii } \\
\text { Automeris zugana } \\
\text { Hylesia } \text { sp. }\end{array}$ \\
\hline & & Coleoptera & Curculionidae & Phelypera distigma \\
\hline Sterculiaceae & Waltheria indica & Lepidoptera & Noctuidae & Diphetera festiva \\
\hline Thymelaeaceae & Daphnopsis americana & Hemiptera & Scutelleridae & Pachycoris sp. \\
\hline Tiliaceae & Apeiba tibourbou & Lepidoptera & Saturniidae & Arsenura batesii \\
\hline Tiliaceae & Luehea candida & Lepidoptera & $\begin{array}{l}\text { Saturniidae } \\
\text { Saturniidae }\end{array}$ & $\begin{array}{l}\text { Arsenura batesii } \\
\text { Arsenura armida }\end{array}$ \\
\hline Trigoniaceae & Trigonia rugosa & $\begin{array}{l}\text { Coleoptera } \\
\text { Hemiptera }\end{array}$ & $\begin{array}{l}\text { Cantharidae } \\
\text { Pentatomidae }\end{array}$ & $\begin{array}{l}\text { Chauliognathus tricolor } \\
\text { Edessa sp. } 2\end{array}$ \\
\hline Ulmaceae & Celtis iguanaea & Lepidoptera & Notodontidae & Polypoetes sp. \\
\hline Ulmaceae & Trema micrantha & Lepidoptera & Saturniidae & $\begin{array}{l}\text { Automeris belti } \\
\text { Automeris metzli }\end{array}$ \\
\hline Urticaceae & Urera longipes & Lepidoptera & Saturniidae & Automeris postalbida \\
\hline Verbenaceae & Citharexylum sp. & Coleoptera & Chrysomelidae & Platyphora bicolor \\
\hline Verbenaceae & Lantana camara & Lepidoptera & Sphingidae & Manduca florestan \\
\hline Verbenaceae & Vitex cooperii & Lepidoptera & Lymantriidae & Rolepa sp. \\
\hline
\end{tabular}

La recolecta manual permitió obtener información sobre la historia natural de los artrópodos recolectados. Los principales grupos recolectados fueron: Lepidoptera (27.47\%), Hymenoptera $(24.40 \%)$ y Coleoptera $(19.03 \%)$; también se recolectaron otros grupos tales como: Hemiptera (7.18\%), Orthoptera (6.64\%) y Polydesmida (3.60\%). Los lepidópteros recolectados manualmente corresponden a estadios larvales; en Hymenoptera la recolecta más frecuentes fue de panales (nidos), adultos y sus estadios; en Coleoptera este porcentaje se refiere a los fitófagos (adultos) y a las larvas y/o adultos que se alimentan de madera, principalmente. El $48.83 \%$ de los organismos recolectados manualmente eran adultos y el $31.40 \%$ correspondió a diferentes estadios larvales. El $20 \%$ restante eran prepupas, pupas, ninfas en diferentes estadios y otros materiales (panal, ootecas, etc.). La recolecta manual se consideró un método efectivo, en el cual se invierte mucho tiempo pero con muy buenos resultados, ya que se obtuvo 557 muestras de 289 especies.

El $25.73 \%$ de las muestras se obtuvo mediante crianza y de éstas, el $98 \%$ pertenecen al orden Lepidoptera, $1.23 \%$ y $0.62 \%$ a los orde- nes Hymenoptera y Coleoptera, respectivamente. La mayor cantidad de muestras del orden Lepidoptera correspondió a estiércol (36.84\%), diferentes estadios larvales (32.51\%), prepupas (17.03\%), pupas (11.76\%) y material asociado como las mudas (1.24\%). Mediante este método se recolectaron muestras provenientes de 117 especies de artrópodos, de las cuales se logró obtener un total de 323 muestras. En general, el orden Lepidoptera se puede criar fácilmente ya que algunos hospederos eran conocidos en el área de estudio y presentaban estadios inmaduros reconocibles e incluso identificables a nivel de especie y tenían un peso fresco relativamente alto. Unos pocos individuos podían completar una muestra, pero como tenían una alta cantidad de agua, fue necesario aumentar el peso fresco recolectado en estos casos (Sittenfeld $e t$ al. 1999). El estiércol de cada estadio larval también se analizó, diversificando el número de muestras de la misma especie.

Con la trampa de luz se recolectó el $18.80 \%$ de las muestras correspondientes a los siguientes ordenes: Lepidoptera (38.13\%), Orthoptera $(25.42 \%)$ y Coleoptera $(19.91 \%)$ y 
otros grupos como Homoptera (5.50\%), Hemiptera $(3.81 \%)$ y Megaloptera $(2.54 \%)$. Todas las muestras correspondieron a individuos adultos. Esta técnica permitió la recolecta de 236 muestras de 164 especies.

Con la trampa de luz fue posible recolectar ambos sexos de la misma especie. Lo anterior aplica para la mayoría de los ordenes, excepto para Lepidoptera donde más del $90 \%$ de los individuos que llegan a las luces son machos. Sin embargo, se recolectaron algunas hembras grávidas de hospederos conocidos que se utilizaron para criar las larvas que eclosionan de sus huevos. De una hembra se pudieron obtener varias muestras, como es el caso de las especies polífagas.

Con las redes (aérea, de golpe y acuática) se recolectaron 132 muestras de 91 especies. El $10.52 \%$ de las muestras, principalmente de los órdenes Orthoptera $(26.50 \%)$, Hemiptera (21.97\%), Coleoptera (16.67\%), Hymenoptera $(9.85 \%)$ y Lepidoptera $(9.09 \%)$. También se utilizaron otros métodos de recolecta de artrópodos $(0.16 \%)$, entre los cuales se encuentra la trampa de foso, con carne o estiércol de caballo como cebos.

En 1997 y 1998, se enfatizó en la recolecta de muestras que resultaron positivas en diferentes ensayos biológicos. El proceso de investigación posterior es lento y por lo general requiere material adicional, para efectuar el aislamiento y caracterización de un compuesto activo. A la fecha los primeros resultados de los bioensayos se han informado para actividad antiparasitaria (Chinchilla et al. 2003).

Este estudio aporta nueva información sobre la recolecta de artrópodos para prospección que puede ser útil para países que cuentan con una alta diversidad biológica, al describir los sistemas de recolecta más exitosos y congruentes con la utilización del recurso de una manera sostenible. Los datos biológicos y químicos constituyeron el valor agregado de la muestra, ya que no es solamente un extracto crudo de material biológico, sino un conjunto de información clave que guía un proceso de investigación y de conservación. Esta información podría ser de importancia para la determina- ción de la fuente del compuesto activo y las condiciones bajo las cuales se produce.

En los estudios de bioprospección de artrópodos se debe evitar la recolecta intensiva durante varios años consecutivos en la misma localidad y procurar la recolecta de la menor cantidad posible de indivuduos, evitando, la alteración de las poblaciones silvestres de artrópodos. Debido a que la dinámica de la mayoría de sus poblaciones no ha sido ampliamente estudiada, es preferible recolectar especies de las cuales se tenga información básica para evitar daños posteriores. Se recomienda minimizar la interferencia en procesos vitales de los organismos tales com reproducción, comportamiento y alimentación. Es necesario escoger la época del año adecuada, permitir el restablecimiento natural de las poblaciones y ser cuidadoso con la extracción masiva de hembras. La bioprospección de artrópodos es posible, si se diseñan prácticas de recolecta que no alteren procesos fundamentales de los organismos, así como interacciones entre organismos en los ecosistemas.

\section{AGRADECIMIENTOS}

Esta investigación fue financiada por el National Institute of Health (Fogarty International Center) No. 5U01TW/CA00312, facilitado por el proyecto NSF DEB 9400829 y DEB 9705072, D. H. Janzen y el proyecto VI 801-96-582 de la Vicerrectoría de Investigación de la Universidad de Costa Rica. Se contó con el apoyo logístico del Área de Conservación Guanacaste, Costa Rica. Un agradecimiento especial a Adrián Guadamuz y Daniel Pérez, asistentes de campo. Un reconocimiento al personal del ACG por su ayuda incondicional durante la fase de campo. Las siguientes personas colaboraron de una $\mathrm{u}$ otra forma con el desarrollo de esta investigación: Winnie Hallwachs, Róger Blanco, Mariano Pereira, Einer Eras, Isabel Salas, Hazel Mora, Felipe Chavarría, Sandy Salas, así como los parataxónomos y "gusaneros" del ACG. Expresamos nuestra gratitud a Gilberth Barrantes y 
Mahmood Sasa por sus comentarios y sugerencias a una versión preliminar del manuscrito y a María Auxiliadora Mora y Diego Loría por su ayuda en el desarrollo de las bases de datos. La identificación de las muestras fue posible gracias a la ayuda de curadores y técnicos del INBio.

\section{RESUMEN}

Desde 1994 hasta 1997, 1800 muestras de artrópodos correspondientes a 642 especies distribuidas en 21 órdenes y 95 familias fueron recolectadas en 21 localidades protegidas del Área de Conservación Guanacaste, para ser estudiadas como fuente potencial de nuevos medicamentos. Las localidades con más recolectas fueron Santa María (231 spp.; 421 muestras), Santa Rosa (110 spp.; 172 muestras), Cacao (98 spp.; 203 muestras) y Pitilla (67 spp.; 79 muestras), siendo la mayor cantidad recolectadas durante la estación lluviosa. El $49.72 \%$ de las muestras recolectadas correspondió al orden Lepidoptera, $15.75 \%$ a Coleoptera, $13.33 \%$ a Hymenoptera, $11.43 \%$ a Orthoptera, $6.75 \%$ a Hemiptera, $3.20 \%$ a Homoptera y $7.89 \%$ a otros grupos. Además, un $54.26 \%$ de las muestras correspondía a individuos adultos, $19.90 \%$ a larvas, $11.20 \%$ a estiércol, $6.46 \%$ a pupas, $6.12 \%$ a prepupas, $2.07 \%$ a ninfas y $3.74 \%$ a otros estadios. Un $44.38 \%$ de las muestras se recolectó mediante recolecta manual, seguido por crianza de insectos (25.73\%), trampa de luz (18.80\%), diferentes tipos de redes $(10.52 \%)$ y otros métodos $(0.16 \%)$. La alta diversidad de los trópicos y la gran abundancia de artrópodos hace de este grupo una fuente potencial de productos farmacéuticos. Sin embargo, se debe evitar la recolecta intensiva en la misma localidad durante años consecutivos (sobre todo hembras), minimizar la interferencia en procesos vitales de los organismos y la dinámica de sus poblaciones. Además se debe recolectar la menor cantidad posible de individuos y escoger la época del año adecuada, para lograr el reestablecimiento natural de las poblaciones.

\section{REFERENCIAS}

Barboza, P. \& D.K. Letourneau. 1988. Novel Aspects of Insect-Plant Interactions. Wiley. $362 \mathrm{p}$.

Chinchilla Carmona, M., M. Herrera Corrales, O.M. Guerrero Bermúdez, A. Jiménez Somarribas, G. Tamayo, A. Sittenfeld Appel, V. Nielsen \& P. Hurtado. 2003. Efecto de extractos de artrópodos sobre la multiplicación del Toxoplasma gondii dentro de macrófagos peritoneales de ratón. Rev. Biol. Trop. 51: 317-320.
De la Fuente, J. 1994. Zoología de Artrópodos. McGraw-Hill-Interamericana, Madrid, España.

Demain, A.L. 1998. Microbial natural products: alive and well in 1998. Nature Biotechnology 16: 3-4.

Dillon, R.J., C.T. Vennard \& A.K. Charnley. 2000. Exploration of gut bacteria in the locust. Nature 403: 851 .

Maes, J.M. 1998. Catálogo de los insectos y artrópodos terrestres de Nicaragua. Vol. 1. Print-León, Nicaragua. $485 \mathrm{p}$.

Mateo, N. 1996. Wild Biodiversity: The Last Frontier? The Case of Costa Rica. The Globalization of Science: The Place of Agr. Res. 73-82 p.

Mateo, N., W. Nader \& G. Tamayo. 2001. Bioprospeting. Encyclopedia of Biodiversity, Volume 1. Academic, New York. pp. 471-488.

Moreno, E. 1997. In search of a bacterial species definition. Rev. Biol. Trop. 45: 753-771.

Nielsen, V. 2003. Métodos para colectar insectos. Rev. Agron. Trop. 33: 59-68.

Obando, V. 2002. Biodiversidad de Costa Rica: estado del conocimiento y gestión. Editorial INBio, Santo Domingo de Heredia, Costa Rica. 17 p.

Rosenthal, J. 2002. Curtain has fallen on hopes of legal bioprospecting. Nature 416, 15 .

Setzer, M.C., D.M. Moriarity, R.O. Lawton, W.N. Setzer, G.A. Gentry \& W.A. Haber. 2003. Phytomedicinal potential of tropical cloudforest plants from Monteverde, Costa Rica. Rev. Biol. Trop. 51: 647674.

Sittenfeld, A. \& R. Villers. 1993. Exploring and Preserving Biodiversity in the Tropics: The Costarrican Case. Current Opinion. Biotechnol. 4: 280-285.

Sittenfeld, A. 1996. Issues and strategies for bioprospecting. Genetic Engineering and Biotechnology UNIDO, Emerging Technology Series 4: 1-12.

Sittenfeld, A., \& A. Lovejoy. 1996. Biodiversity Prospecting Frameworks: The INBio Experience in Costa Rica. Protection of global biodiversity: Converging Strategies pp. 223-244.

Sittenfeld, A. , G. Tamayo, V. Nielsen, A. Jiménez, P. Hurtado, M. Chinchilla, 0. Guerrero, M. A. Mora, M. Rojas, R. Blanco, E. Alvarado, J.M. Gutiérrez, \& D.H. Janzen. 1999. Costa Rican international cooperative biodiversity group: using insects and other arthropods in biodiversity prospecting. Pharm. Biol. 37(Suppl.): 55-68. 
Sittenfeld, A. \& A. Lovejoy, 1999. Managing bioprospecting and sustainable use of biological diversity. Managing Agricultural Biotechnology-Addressing Research Program Needs and Policy Implications for Developing Countries. pp. 92-101.

Sittenfeld, A., L. Uribe-Lorío, M. Mora, V. Nielsen, G. Arrieta \& D.H. Janzen. 2002. Does a polyphagous caterpillar have the same gut microbiota when feeding on different species of food plant? Rev. Biol. Trop. 50: 547-560.

Slater, J.A. \& R. M. Baranowski, 1978. How to know the true bugs (Hemiptera - Heteroptera). Brown, USA. $256 \mathrm{p}$.

Spiteller, D. S., K. Dettner \& W. Boland. 2000. Gut bacteria may be involved in interactions between plants, herbivores and their predators: microbial biosynthesis of $\mathrm{N}$ - acylglutamine surfactants as elicitors of plants volatiles. Biol. Chem. 381: 755-762.

Tamayo, G., W.F. Nader \& A. Sittenfeld. 1997. Biodiversity for the Bioindustries. Biotechnology and Plant Genetic Resources: Conserv. and use. pp. 255-279.

Ten Kate, K. 2002. Science and the Convention on Biological Diversity. Science 295: 2371-2372

Thayer, A.M. 1998. Living and loving life sciences. Chem. \& Eng. News 76: 16-24.

Weiss, C. \& T. Eisner. 1998. Partnerships for value-added through bioprospecting. Technol. in Soc. 20: 481498. 